\title{
The automatic definition and generation of axial lines and axial maps
}

\author{
Michael Batty, Sanjay Rana \\ Centre for Advanced Spatial Analysis, University College London, 1-19 Torrington Place, \\ London WC1E 6BT, England; e-mail: m.batty@ucl.ac.uk
}

Received 5 September 2002; in revised form 3 November 2003

\begin{abstract}
Space syntax is a technique for measuring the relative accessibility of different locations in a spatial system which has been loosely partitioned into convex spaces. These spaces are approximated by straight lines, called axial lines, and the topological graph associated with their intersection is used to generate indices of distance, called integration, which are then used as proxies for accessibility. The most controversial problem in applying the technique involves the definition of these lines. There is no unique method for their generation; hence different users generate different sets of lines for the same application. In this paper we explore this problem, arguing that to make progress, there need to be unambiguous, agreed procedures for generating such maps. The methods we suggest for generating such lines depend on defining viewsheds, called isovists, which can be approximated by their maximum diameters, these lengths being used to form axial maps similar to those used in space syntax. We propose a generic algorithm for sorting isovists according to various measures, approximating them by their diameters and using the axial map as a summary of the extent to which isovists overlap (intersect) and are accessible to one another. We demonstrate our techniques for the small French town of Gassin used originally by Hillier and Hanson in a 1984 book to illustrate the theory, exploring different criteria for sorting isovists, and generating different axial maps by changing the level of resolution.
\end{abstract}

\section{Introduction: the problem}

Space syntax provides a method for partitioning a spatial system into relatively independent but connected subspaces so that the importance of these subspaces can be measured in terms of their relative nearness or accessibility (Hillier and Hanson, 1984). It is similar to a wide class of models for measuring spatial interaction, developed over the last fifty years as part of social physics, which derive relative accessibility from the underlying graphtheoretic structure of relations, usually based on the Euclidean distances between small areas (Wilson, 1998). It differs from this class, however, in three significant ways. First, the subspaces or small areas which compose the basic representational elements in space syntax are ill defined. The spatial elements used are not directly observable and measurable and, although they depend upon the geometric properties of the space, there is no agreed or unique method for their definition. Second, spaces are not collapsed to nodes or points but are first defined by lines which are then considered as nodes. Third, the relations between these components or nodes are defined in terms of their topology and, although Euclidean distance is implicit, relations are measured in binary terms - whether they exist or not.

In this paper we will focus entirely on the first problem which involves defining the spatial components used in subsequent relational analysis. We will introduce methods which resolve the problem of deriving a unique set of elements, thus enabling their automatic definition. These methods extend quite naturally to the second and third problems in that representing lines as nodes is no longer necessary. The method we introduce suggests that the relative importance of lines associated with subspaces is

- Current address: Department of Geography, University of Leicester, Bennett Building, Leicester LE1 7RH, England; e-mail: sr115@le.ac.uk 
often an approximate function of their length. In short, we introduce a method which collapses all three stages into one, although it is still possible to use the elements we generate to conduct conventional space syntax analysis thereafter. In later papers we will address the second and third problems, although the work we embark upon here implies the need for a much more fundamental theory of morphology. It is this, we argue, that should constitute the long-term agenda for space syntax.

Space syntax begins with an exhaustive decomposition of the space into mutually exclusive subspaces which are assumed to be convex. In the original formulation, various standard graph-theoretic relations based on the adjacency of these subspaces were proposed, but methods based on such adjacencies have hardly been developed at all. Instead, what is usually done is to link these subspaces using straight lines or axes which intersect with one another to provide a system of 'axial lines' which is called an 'axial map'. This is an approximation to the convex geometry of the system, but with only a loose connection between axiality and convexity. Subsequent analysis simply takes these axial lines; as nodes of a graph, with their intersections constituting relations between these nodes, and derives standard distance measures which, when summed at each node, provide measures of accessibility for any line to all others. The focus on axiality implies that direction and orientation are important to the analysis and this has implications for the use of this kind of analysis in studying movement. More recent work has introduced the concept of the 'viewpoint' associated with each axial line; in some interpretations, axial lines are associated with lines of sight, or at least lines of unobstructed movement through the space. These latter developments do not map easily onto the basic defintion of lines as measures for summarizing space, but they have propelled the analysis towards associating axial lines with transport and traffic. In traditional analysis the nodes in graphs based on relationships between elements in a map are associated with densities, intensities, and potential development at point locations, but in the case of space syntax these same nodes imply movements over a line, which reveals a more complicated definition of density.

Two issues make space syntax controversial. First, although there is some guidance as to how axial lines might be generated, much is left to the user's intuition and graphic dexterity. Thus there is always the suspicion that each example cannot be replicated by a different user in a different time at a different place. Desyllas and Duxbury (2001, page 27.6) make the point when they say: "the ... axial map cannot provide researchers with reliable and comparable results." Peponis et al (1998, page 560) argue that objectivity in the process of generating axial lines can only arise "from the rigor and repeatability of the procedures used to generate them." Second, the twist that is occasioned by treating lines as nodes is counter to the way social physics and transportation analysis have developed, where density and volume of movement are intrinsically associated with point locations, not geometrically artificial lines defined by users where length (and hence cost and travel time) is ignored. Many of these problems arise from the fact that methods and measures for implementing the theory have not been well formulated. In fact, from the variety of publications over the last two decades it is clear that multiple space syntaxes exist and that there is no standard way of engaging in this analysis. What we will do in our paper is to lay bare the assumptions and, in doing so, propose procedures for generating axial lines and axial maps which lead to unique and reproducible results. The appropriateness of our methods must be judged on the assumptions made in adopting a particular procedure in the first place. This is what the theory and its methods currently lack. We believe that, by introducing automatic methods, space syntax has a chance to relate to more mainstream ideas in morphology and social physics, thus widening its appeal to disciplines beyond architecture. 
We will begin by discussing the key problems of partitioning a spatial system into convex subspaces and describing this by axial lines. It has been known for a long time that there is no formal procedure for generating a unique partition of a two-dimensional space into convex polygons (O'Rourke, 1987). We will also address the ways in which axial lines relate to convexity, clarifying how and why it is necessary to consider viewpoints or centroids which link areas to lines. We then state the essence of our argument which is based on unambiguously specifying the conditions that an axial line must meet, and then deriving a procedure for ordering and sorting the axial lines in terms of their relative importance. What this shows is that there may be many different conditions which specify the line and many different kinds of ordering which generate their relative importance to one another. Our method, in fact, is based on defining viewsheds, called isovists, and generating different axial lines from different properties of these viewsheds. This implies different kinds of syntax. Although we first hone our ideas on the basic 'T-shape' used in many previous papers, we illustrate these ideas with the original example used by Hillier and Hanson (1984) of the small French town of Gassin. This immediately reveals that our methods generate different results from those which were originally derived manually and intuitively. We then illustrate how changes in scale or resolution affect the number and form of these isovists (and hence axial lines), indicating how important is the representation of the space in raster/vector terms in establishing a degree of invariance for this style of analysis. We conclude by anticipating further research which we have underway and how this might lead to generic theories of urban morphology. Ways in which we have automated these procedures based on the well-known desktop geographic information system (GIS) ArcView are available at http://www.casa.ucl.ac.uk/sanjay/software_isovistanalyst.htm.

\section{Defining space: convex partitions, axial lines, and isovists}

\subsection{Generating convex spaces}

Although most applications of space syntax have emphasized how the areas constituting rooms in buildings and street systems between urban parcels can be simplified by using axial lines - lines of unobstructured movement - the theory as developed by Hillier and Hanson (1984) defines two complementary approaches to spatial definition: convexity which emphasizes the two-dimensional features of the system and axiality which emphasizes the one-dimensional. We will begin by briefly summarizing these and then turn to a more recent development in the theory which incorporates ideas concerning viewpoints, viewsheds, or isovists as defined by Benedikt (1979).

Hillier and Hanson (1984) argue that the partition of space should meet an implicit condition of enclosure that they assume is met by geometrically convex subspaces. They define a convex map as: "the least set of fattest spaces that covers the system" (page 92), and they continue by suggesting an algorithm for manually constructing such a convex map: "Simply find the largest convex space and draw it in, then the next largest, and so on until all the space is accounted for" (page 98). However, given the continuity of space, such partitioning is not well defined. Even if there is a minimum number of subspaces which are convex, these cannot be found and, in any case, the criterion for what is a 'fat' convex space is never defined. In fact, although space syntax has largely ignored these formal considerations of convexity in practical applications, some progress has been made in defining what Peponis et al (1997) call "informationally stable spaces" which do meet conditions of convexity. They demonstrate that there is no partition which gives a minimum number of subspaces whose convexity is unique as we illustrate in figure 1(a) (over). But they suggest that it is possible to generate a larger number of convex spaces which they call an s-partition. This partition is made at points of discontinuity which define the edges or faces of the space where there is a change in 


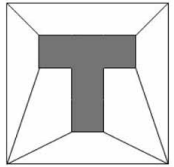

(a)

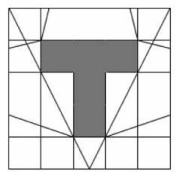

(c)

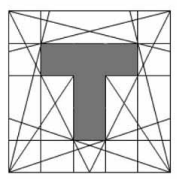

(e)
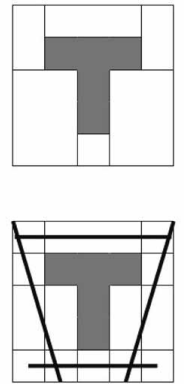

(d)

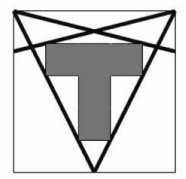

(f)
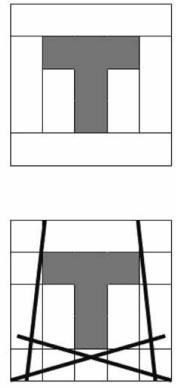

(b)

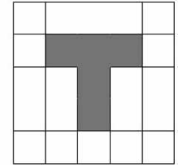

Figure 1. Convex sets, partitions, and axial lines for the basic T-shape.

the number of surfaces which come into view as the partition is crossed. We show this in figure 1(b). They then extend this criterion by defining e-partitions (of which the s-partition is a subset) - lines between vertices defining the faces or edges of the form in which other faces or edges gradually or immediately become visible as the lines are crossed. These are extendible diagonals and we show such e-partitions and their e-spaces in figure 1(c). Details of the actual procedure for their definition are given in the Spatialist software used to generate these and in papers by Peponis et al $(1997 ; 1998)$. The advantage of these types of decomposition is that they are unique and informationally stable in the sense that they reflect significant visual (geometric) thresholds which can then be subjected to standard relational analysis.

\subsection{Generating axial lines and axial maps}

The dominant approach in space syntax involves representing these convex polygons (or their approximation) by using straight lines which imply unobstructured movement between spaces. The usual approach in spatial analysis, however, is to approximate subspaces by points or centroids and then to conduct analysis on networks of relations between these points. In contrast, space syntax defines aggregations of subareas by lines, and it is the procedure for doing this that is the most controversial aspect of the analysis. Hillier and Hanson (1984) define an axial map as: "the least set of such straight lines which passes through each convex space and makes all axial links" (page 92), and they define the procedure for doing this in analogy to that for the convex map by: "first finding the longest straight line that can be drawn ..., then the second longest, and so on until all convex spaces are crossed and all axial lines that can be linked to other axial lines without repetition are so linked" (page 99). There are many problems with this procedure, not least the fact that, once convex spaces are 'covered' or crossed by axial lines, the aggregate of the space crossed is no longer convex; otherwise it would have been defined as such in the first instance. A second problem relates to the fact that, for spaces to be related, axial lines must intersect and this means the axial map must be strongly connected. All subspaces might be crossed without the axial map being connected in this way. So to avoid such problems, an (arbitrary) criterion of making each axial line link to at least one other is imposed. Because a unique set of least, fattest convex spaces cannot be defined, it is thus impossible to automate the construction of an axial map. As the procedure is left to 
the user, the biggest problem is controlling for the number of axial lines as this is central to the accessibility values which are subsequently computed and used to index the importance of each line. In figure 1(d) we present intuitively derived axial maps which cover the s-spaces in figure 1(b), where it is clear that the second map might be said to cover the space 'less comprehensively' than the first, although the first has slightly 'longer lines'. It is problems such as this that we seek to address in this paper.

The all-line axial map, first defined and thence published by Penn et al (1997) but used extensively by Hillier (1996) in his second book, consists of all possible lines that link vertices defining differences in orientation between faces as well as all extensions of faces to meet other faces. There is an added constraint that such lines must pass freely through space which is unobstructed. Peponis et al (1998) present three different methods. These all begin with the all-lines map illustrated in figure 1(e) and in each case, they reduce the number of lines in this map while meeting different criteria for covering the convex spaces. One of these methods is particularly straightforward, being based on ranking the number of diagonals in the all-lines map with respect to the number of s-partitions that each diagonal crosses. The diagonal with most crossing points becomes the first axial line. This and the associated s-partitions are then removed, the remaining diagonals reranked, the largest chosen, the set of diagonals and s-partitions reduced further, and so on until all partitions have been crossed. This method leads to the axial map in figure 1(f) which is closer to but still somewhat different from the first map in figure 1(d). These methods show promise but, as they depend on the vertex geometry of the original plan or layout, they remain restrictive in terms of where lines can be drawn.

\subsection{Viewsheds: isovists and isovist fields}

The third approach which has emerged in the last decade is rather different, although there are important antecedents reflected in the work of the Hillier and Peponis groups. This approach depends not on simplifying morphology as a map which covers a subdivision into convex spaces but on describing the morphology in terms of individual points which in themselves cover areas of the space. The shift is thus from area to point analysis, although still in terms of describing the morphology of these points by lines which cover space as sources for lines of unobstructed movement or lines of sight. How far one can see or move thus becomes the key criterion for definition. The object which defines this approach is the viewshed, visual field, or isovist constructed around a given point in the space, with its generalization to an isovist field which describes what is contained within each viewshed or isovist at every point in the space. It is possible to approximate everything contained in the space if the viewpoints are chosen regularly at a sufficiently fine level of resolution. Isovists might be defined for any of a very wide number of measures: for example, distance, area, or perimeter seen from a point, or measures related to these, as well as any other objects within the viewshed. How far or how much one can see or access in an unobstructed way is the usual measure, but the idea is easily generalized for capturing a very wide variety of features and objects which fall within a specific viewshed. Although space syntax did not originally embrace the idea of an explicit viewpoint or viewshed, in one sense it is deeply embedded in the theory. All the relational analysis between convex spaces and axial lines from which the importance of these spaces or lines is derived is based on the notion that what is important is the number of different things that might be seen or accessed from a particular space or line, not the actual distance to these different things. In fact, much of the research just summarized on s-partitions and e-partitions as well as on the all-line map is predicated on the notion that points and lines where viewsheds significantly change are key elements in simplifying the space. 
Viewshed analysis has been widely developed in landscape studies and is integral to GIS (Rana, 2002), but there has been very little research on its application to urban areas, with one or two notable exceptions. Benedikt (1979), in a pioneering paper on urban viewsheds, adopted the term isovist from Tandy (1967) who had used it to describe views in landscapes. Until quite recently, Benedikt's ideas were developed in a somewhat ad hoc way by the space syntax community, with the clearest statements in Hillier's (1996) book, and in the Spatialist software developed by the Peponis group. Recently Batty (2001), Dalton and Dalton (2001), Ratti (2002), and Turner et al (2001) have all suggested that isovist fields represent an alternative way of simplifying urban and building morphologies by using ideas from visibility graphs, agent-based modeling, ray tracing, and image analysis. Software such as Depthmap from Turner (2001), OmniVista from Dalton and Dalton (2001), and Fathom from Intelligent Space (2002) have appeared which make the generation of isovist fields automatic. There is a strong implication that the isovist field idea is preferable to the definition of axial maps because of its inherently well-defined nature and consistent replicability. Desyllas and Duxbury (2001) go further and suggest that isovist field statistics represent more appropriate ways of measuring accessibility in urban areas than axial maps because isovist fields provide better correlations with observed movements and the problem of averaging observed density volumes along a line is avoided (Turner and Penn, 1999).

The advantage of thinking about space in terms of isovists and isovist fields is that, for a sufficiently fine level of resolution, there is a complete description of how far one can see or move from every point in the space. Moreover, each viewpoint is associated with a space - the viewshed-which can be approximated by a line which spans the space, somewhat like a diameter. The major difference from space syntax is that isovists are not in general convex spaces although it is possible to define a convex core to each (Hillier, 1996). Although the rest of this paper will be concerned with extracting axial lines from isovists, we must anticipate these to show how they compare with those already described. One method is: first find the isovist with the longest diameter, select this as the first axial line, and reduce the space to be considered next by subtracting the isovist associated with this first line. Then find a viewpoint in the remaining space which generates the next longest line, select this, and reduce the space further by subtracting that isovist from the active space. Continue in this manner until all the space has been covered. The set of lines extracted will constitute the axial map. These may not always be connected, but their isovists will be, because all the space has been systematically tessellated into dominant isovists whose contiguity ensures an exhaustive partition of the space.

In this context, it is the isovist itself which is used to capture information about the space, and its diameter is used only to approximate its span. If we use the isovist to capture activity of varying density, then the lines which are selected and their order need not follow the rule of selection from longest to shortest. Thus length need not be the sole criterion for spatial ordering. In one sense, what this method does is to side step the convexity problem by arguing that what is contained within the isovist must be used to order space. If this is defined to be the size of the convex core of the isovist, then this would orient the problem back towards traditional space syntax. In figures 2(a) to 2(d) we define four isovists for key corner points in our T-shape. If we then use the basic criterion that the longest straight line in each isovist is to be used to order the space, it is clear that there are multiple isovists lying along the left-hand corners of the $\mathrm{T}$ which all generate the same longest line.If we reduce the space by the isovists associated with this line, then the isovists associated with the right-hand corners of the $\mathrm{T}$ generate a similar line on the right-hand side. It is intuitively obvious that the resultant $\mathrm{V}$ shape within which the T sits defines two axial lines for isovists which cover all the space. Thus the solution produced using this method which is shown in figure 2(e) has similarities 


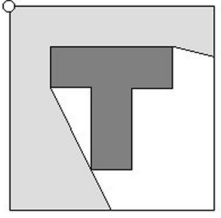

(a)

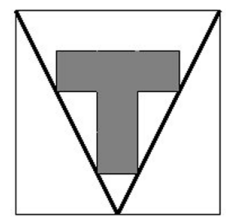

(e)

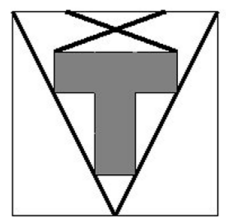

(f)

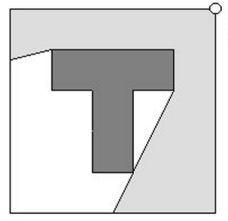

(b)

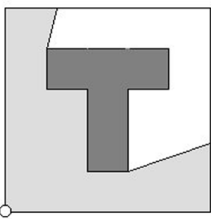

(c)

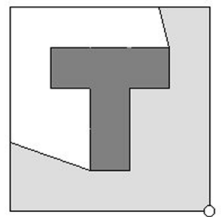

(d)

Figure 2. Key isovists and related axial lines for the basic T-shape.

with one of those already generated using more conventional analysis. There are many differences but, before we develop the method further, we must briefly explain what happens next in terms of space syntax, once an axial map has been produced.

\subsection{Relational analysis of convex spaces and axial lines}

Most of the effort in space syntax has not been in generating axial lines or maps but in deriving and interpreting relationships between the lines that constitute such maps through the number of changes of direction or the number of paths between lines defining the spaces which constitute the system. In essence, relations between convex spaces can be measured in terms of whether or not common adjacencies exist, or between axial lines in terms of whether or not intersections with other lines exist. If we call the spaces or lines $i$ and $j(i, j=1,2, \ldots, N)$ and the number of adjacencies or intersections $k$ and $l(k, l=1,2, \ldots, M)$, the matrix $\mathbf{A}=\left[A_{i k}\right]$ defines the existence $A_{i k}=1$ (or not, $A_{i k}=0$ ) of adjacencies and intersections $k$ that are associated with spaces or lines $i$. The matrix of relations between pairs of spaces or pairs of lines is computed as $R_{i j}=\sum_{k} A_{i k} A_{j k}$ or $\mathbf{R}=\mathbf{A} \mathbf{A}^{\mathrm{T}}$, from which we define the binary matrix $\mathbf{L}$ as $L_{i j}=1$ if $R_{i j}>0(i \neq j)$, or $L_{i j}=0$ otherwise. From the graph implied by this symmetric matrix, all relations are derived as functions of shortest paths. The number of paths of length $t+1$ from $i$ to $j$ is defined from the recurrence $L_{i j}^{t+1}=\sum_{l} L_{i l}^{1} L_{l j}^{t}(t=1,2, \ldots, N)$ with the shortest path between $i$ and $j$ given by the matrix $D_{i j}=t+1$ if $L_{i j}^{t+1}>0$ and $L_{i j}^{t}=0(t=1,2, \ldots, N)$.

From the shortest path matrix, distances associated with each node (space or line) are computed as sums of indegrees or outdegrees. That is, a typical total distance (or depth) for a line $i$ is computed as $D_{i}=\sum_{j} D_{i j}$ which is also proportional to the accessibility or integration of the line. In fact, integration is usually taken as the inverse $D_{i}^{-1}$ and it is these values that are compared with densities or volumes of movement associated with the axial lines. Although most applications of space syntax begin after these integration values have been defined, there are many issues to be clarified concerning the appropriateness of this relational analysis. For example, it is well known that, for any system of relations defined on two sets, there are always dual 
problems which consist in interpreting relations between one set of elements through the other and vice versa (Batty and Tinkler, 1979). In this case, there is a dual problem where the matrix of relations is computed from $\hat{\mathbf{R}}=\mathbf{A}^{\mathrm{T}} \mathbf{A}$. In the case of axial maps, the relations would generate accessibilities for the points of intersection, not for the lines themselves. In fact, in early studies of urban morphology, Atkin (1974) developed an approach called $Q$-analysis which sought to examine urban structure in terms of these duals (Atkin, 1974). Such extensions open up an entirely new domain of research in space syntax which we will explore in a later paper, but, for now, we will refocus our interest on the extraction of axial lines from isovists and axial maps from isovist fields.

\section{Axial lines from isovists and isovist fields}

\subsection{Definitions, properties, and measures}

An isovist is defined as the space which can be directly accessed from a specific viewpoint. This might be the space which can be seen by an observer and is often taken (as it is here) as the entire space viewed when the observer moves through $360^{\circ}$ or $2 \pi$ radians. But it might also be the space through which an observer can transport himself or herself without geometric obstruction. In space syntax most applications have been restricted to architectural and urban systems at scales where lines of sight are important although, in principle, these ideas can also apply to morphologies where sight and vision are not relevant. The focus on scales where vision is relevant, however, is significant because considerable work in space syntax appeals to what and how far one can see as being instrumental in the molding of the urban fabric. With this in mind, an isovist is a nonconvex space arrayed around a viewpoint $i$ which we illustrate for a small urban streetscape in figure 3(a) (the viewpoint is indicated by a dot). The space is a polygon which in digital applications is approximated by a raster whose points are also illustrated in figure 3(a). The grid points in this raster are typically other

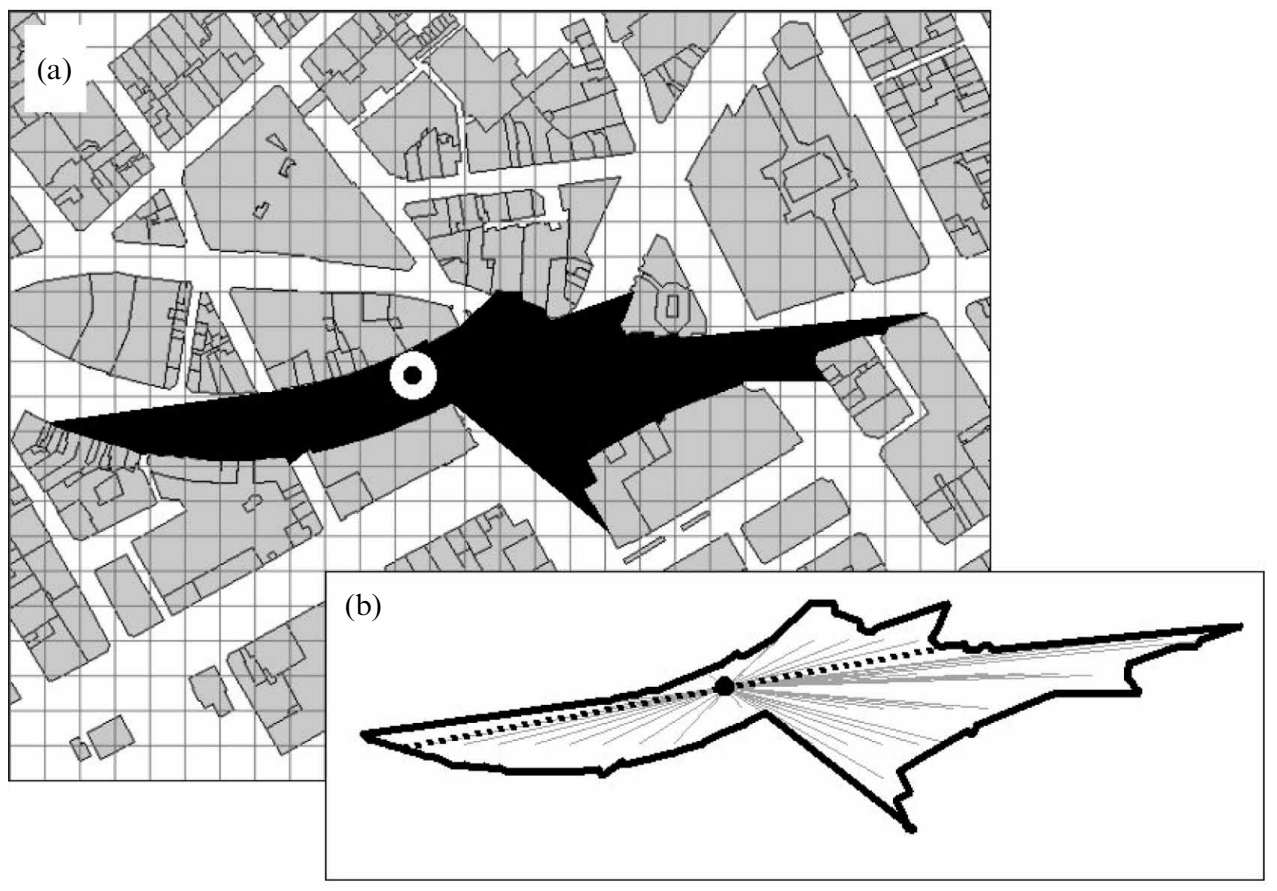

Figure 3. Isovist resolution and maximum diametric length. 
viewpoints for which isovists can also be defined; measures of the shape or what is contained within each isovist are then used to define various isovist fields which are in themselves measures of the morphology of the entire space.

Although our central concern is not with computational issues per se, the question of the scale of resolution used to define isovists is important. As figure 3(a) reveals, the viewpoints are approximated by a raster and the isovist is defined by relating the raster points within the isovist to the viewpoint. Three variants have been used and all these involve tracing rays in circular rotation around the viewpoint and measuring the intersection of these rays with the raster points. The method in the ArcView GIS variant used for the public domain code developed here by Rana (see http://www.casa.ucl.ac.uk/sanjay/ software_isovistanalyst.htm) uses rays but then defines polygons from intersections of the rays with building outlines. A second method used here for the trials in the next section, developed by Batty (2001) using agent-based technology, involves moving agents along rays, measuring properties of the isovist as they travel. This method developed in StarlogoT is by far the fastest of any to date being implemented on a pseudo-parallel processor. The third method, developed by Turner (2001), uses the grid points as nodes in a graph which spans the entire space and enables rapid measures of neighborhood and convexity to be calculated in software called Depthmap. The ray and agent tracing is indicated in figure 3(b), where the rays shown also illustrate all the graph links for the node in question.

There are many geometric measures which can be computed for any isovist which when developed for an entire field of viewpoints, constitute isovist fields. There are one-dimensional measures based on distance and two-dimensional measures based on area and orientation and several measures derived from this geometry can be defined to indicate degrees of compactness, convexity, and circularity. Measures where the isovist is used as a container to collect information about other characteristics of the environment can also be defined, although these depend upon specific associations with activities such as population and other densities. In fact, one of the limitations of space syntax has been an absence in using its spatial aggregations to capture anything other than geometric characteristics and movement. Once such measures have been defined, however, various moments (in particular, means and variances) can be calculated for individual isovists where measures such as distance vary with the isovist and/or across viewpoints which measure variations in the isovist field. For a typical system defined by $n$ viewpoints, we will index a specific viewpoint in the range $i$, $j=1,2, \ldots, n$. The viewpoints $j\left(j=1,2, \ldots, n_{i}\right)$ within an isovist located at viewpoint $i$ are defined by the neighborhood $\Omega_{i}$ which consists of $n_{i}$ viewpoint cells. The basic measures are thus distance, $d_{i j}$, from the core viewpoint $i$ to $j$ and the orientation of the ray associated with this distance, which is $\theta_{i j}$.

The key measure in extracting axial lines as approximations to isovists is based on the diametric length, defined as $\Delta_{i(j k)}=\left(d_{i j}+d_{i k}\right)$, where $\left|\theta_{i j}-\theta_{i k}\right|=\pi$ and $j \neq k$. The relevant values of this measure are its minimum and maximum, given as

$$
\begin{aligned}
& \Delta_{i}^{\min }=\min _{j k}\left\{d_{i j}+d_{i k}\right\}, \\
& \Delta_{i}^{\max }=\max _{j k}\left\{d_{i j}+d_{i k}\right\}, \quad \text { where }\left|\theta_{i j}-\theta_{i k}\right|=\pi, \quad \text { and } j \neq k .
\end{aligned}
$$

Hereafter we refer to the maximum diametric length, $\Delta_{i}^{\max }$, as the 'diameter' of the isovist which we show by the dotted line in figure 3(b). This defines the longest straight line across the isovist, which can be thought of as a maximal spanning distance. Other key measures are the minimum distance and the maximum distance, defined, respectively, as

$$
d_{i}^{\min }=\min _{j}\left\{d_{i j}\right\}, \quad d_{i}^{\max }=\max _{j}\left\{d_{i j}\right\},
$$


with their mean and variance as

$$
\bar{d}_{i}=\sum_{j \in \Omega_{i}} \frac{d_{i j}}{n_{i}}, \quad \sigma^{2}\left(d_{i}\right)=\left[\sum_{j \in \Omega_{i}}\left(\frac{d_{i j}-\bar{d}_{i}}{n_{i}}\right)^{2}\right]^{1 / 2} .
$$

Means and variances of the diametric lengths are not stated as we will not use them in the subsequent analysis although they are computed by the GIS extension to ArcView. A related space exploited by Hillier (1996) associated with any isovist is its convex core and here we note that the minimal convex core is the circle traced out by computing the coordinates around the viewpoint $i$ from $\pi\left(d_{i}^{\min }\right)^{2}$. We show this core as the larger circle based on the viewpoint at the smaller circle in figure 3(a).

When we compare distances we move the observer $m$ times, incrementing the arc each time by $\theta=2 \pi / m$ radians. The means and variances of these angles are not meaningful, but we can compute a mean distance weighted by its orientations and its variance as

$$
d(\theta)_{i}=\frac{\sum_{j \in \Omega_{i}} \theta_{i j} d_{i j}}{\sum_{j \in \Omega_{i}} d_{i j}}, \quad \sigma^{2}\left[d(\theta)_{i}\right]=\left[\frac{\sum_{j \in \Omega_{i}}\left(\theta_{i j} d_{i j}-\theta_{i}\right)^{2}}{\sum_{j \in \Omega_{i}} d_{i j}}\right]^{1 / 2} .
$$

Area and perimeter computations are straightforward although this requires the end points of each ray to be ordered around the circle of revolution where $j=1$ is associated with $\theta, j=2$ with $2 \theta$, and so on. Defining the radial distance for each ray as $r_{i l}$, the area and perimeter are given, respectively, as

$$
a_{i}=\frac{1}{2} \sin \theta \sum_{l \in \Omega_{i}} r_{i l}^{2}, \quad p_{i}=\sum_{l=1}^{m}\left[\left(r_{i l+1} \sin \theta\right)^{2}+\left(r_{i l}-r_{i l+1} \cos \theta\right)^{2}\right]^{1 / 2} .
$$

There are several derived statistics useful for measuring the difference between actual and ideal geometric shapes. We define three which all have values of 0 for a straight-line shape and 1 for a circle: compactness, $\Gamma_{i}$, the ratio of average to maximum radial distance; convexity, $\Psi_{i}$, the ratio of idealized circular to perimeter radius; and circularity, $\Theta_{i}$, the ratio between actual and idealized circular area. A fourth, centrality, $\Phi_{i}$, is a measure of drift or displacement between the centroid of the isovist $\left\{x_{i}, y_{i}\right\}$ and its end viewpoints $\left\{x_{j}, y_{j}\right\}$. These measures are defined, respectively, as

$$
\left.\begin{array}{rl}
\Gamma_{i} & =\frac{\bar{d}_{i}}{d_{i}^{\max },} \quad \Psi_{i}=\left(\frac{a_{i}}{\pi}\right)^{1 / 2} /\left(\frac{p_{i}}{2 \pi}\right), \quad \Theta_{i}=\frac{a_{i}}{\pi \bar{d}_{i}^{2}}, \\
\Phi_{i} & =\left[\left(\frac{\sum_{j \in \Omega_{i}} x_{j}}{n_{i}}-x_{i}\right)^{2}+\left(\frac{\sum_{j \in \Omega_{i}} y_{j}}{n_{i}}-y_{i}\right)^{2}\right]^{1 / 2}
\end{array}\right\} .
$$

All these measures in equations (1)-(6) can be generalized to form different isovist fields and various moments can be computed for subsequent statistical analysis. Isovist fields also have important surface properties which can be exploited to identify various visual thresholds as can be seen in the subsequent examples in this paper as well as in previous published work (Batty, 2001; Turner et al, 2001). These properties have been exploited in landscape analysis (Llobera, 1996; Rana and Morley, 2002), but in this paper we will simply note these and their relationship to mainstream image and pattern analysis. Indeed, there are alternative approaches which suggest that there are many other kinds of axial geometry that might be used to summarize viewsheds. For example, the medial axial transform has been widely exploited in the analysis of shape within biology, and properties of the isovist 
surfaces themselves with respect to their slope and shape can be used to simplify the structures of the fields that they define (Blum and Nagel, 1978; Ratti, 2002). In a related paper, Carvalho and Batty (2003) explore one such extension.

\subsection{Algorithms for generating axial lines}

We consider that the axial line associated with an isovist is the maximum diametric length $\Delta_{i}^{\max }$ defined in equation (1). As we have already noted in discussion of axial lines from isovists for the T-shape in figure 2, this diameter is not associated with a single isovist, for there are an infinity of points along its length from which an appropriate isovist can be generated. As axial lines are used to approximate areas, it is always necessary to select such a line with respect to some areal or other independent measure of an isovist. There must be some way of selecting a unique viewpoint (and hence a unique isovist) and, in this way, the chosen diameter becomes a line uniquely associated with a particular isovist. This is very much in the spirit of space syntax, for axial lines are always associated with spaces which they are designed to link and span. Thus their definition from isovist spaces must always relate what is in the space-its area or some other measure-to the way it is approximated by a line. Therefore, the infinity of points on such a line is never at issue and, if there are ties to be broken, this must be accomplished with respect to some other isovist measure.

There are two variants of the general procedure. The first is based on selecting a longest line and then selecting a point on the line which is associated with an isovist (for which this line is longest) but breaking the tie with other points according to some other measure for which that isovist is maximal. The second method consists selecting the viewpoint of an isovist for which a measure is maximal and then generating its longest line. The first method focuses on longest lines and chooses where they are rooted in viewpoints according to area. The second chooses the largest area, say, and then generates an appropriate line. The first method gives precedence to longest lines, the second to largest areas and then generates the line. Both are part of a generic algorithm which we describe as follows. The algorithm, which we illustrate in figure 4, works by

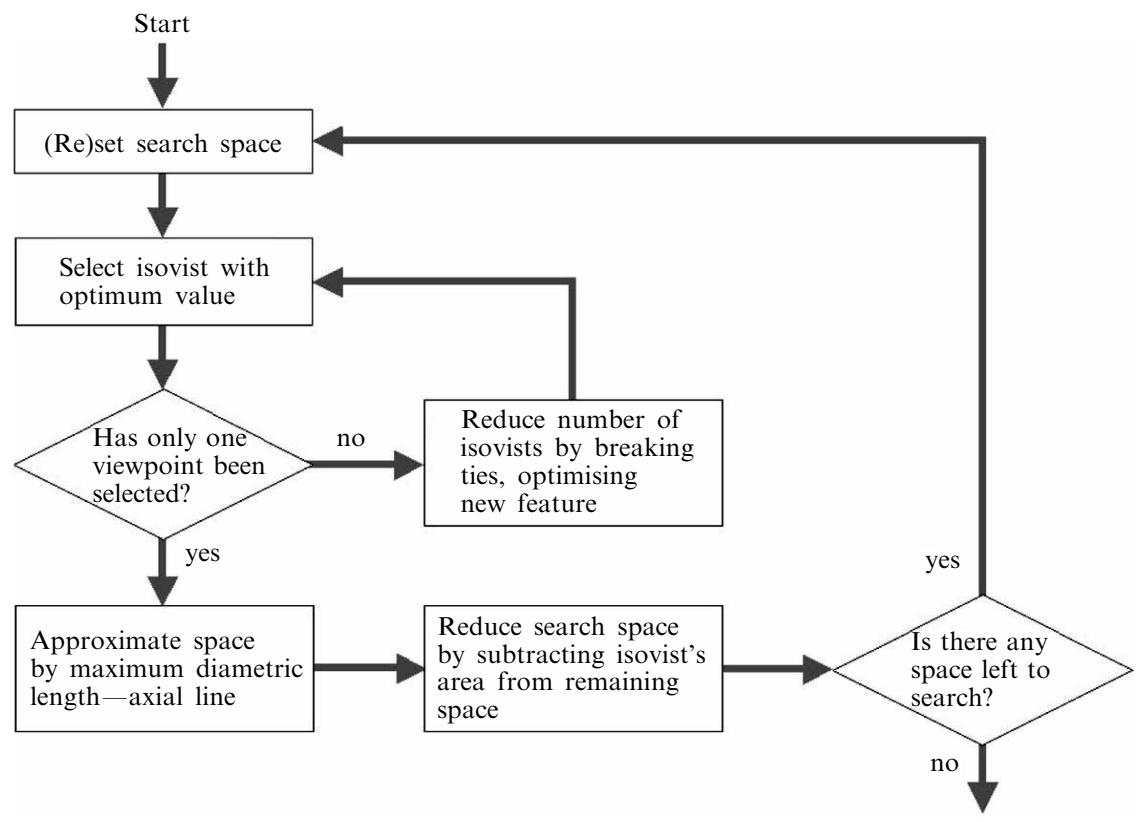

Figure 4. Generic algorithm for sorting isovists and generating axial maps. 
selecting some attribute or measure of the isovist which is to be optimized. It begins by selecting the isovist which meets this criterion of optimality and assumes that the space taken by this isovist is dominant. It selects the axial line-the maximum diametric length - associated with this isovist. The next isovist chosen cannot be rooted within the isovist already chosen because that isovist dominates. To make sure that this cannot happen, the space available for searching for the next isovist is reduced by subtracting the first isovist for the entire space. A second isovist which meets the criterion for optimality is then chosen in the reduced space, its axial line selected, and the space further reduced. This process continues until all the space has been covered by isovist selection. At that point, the axial map that has been generated is simply the dominance ranking of the isovists.

The first method, which starts with the longest lines, is essentially one where the length of line is being optimized. The longest line is chosen and any ties (of which there are many) for isovists associated with this longest line are broken using some other criterion such as largest area. In this way, lines of smaller and smaller length but as long as possible are generated. The second method starts with the isovist associated with the largest area. If there are ties, then these might be broken using the longest line or some other criterion. What this method generates are isovists with smaller and smaller areas. We illustrate the axial lines generated for the T-shape in figure 2(f) where we begin with the isovists covering the largest areas around the $\mathrm{T}$ and proceed in the manner just outlined, reducing the space each time. In essence, this method is not dissimilar from that suggested by Hillier and Hanson (1984) for manual definition of axial lines and convex spaces. It is a very strict ranking of lines or spaces where a larger space or longer line completely dominates the selection of the next space or line. In this sense, dominance is a local criterion and the heuristic is locally optimal. Space syntax has never sought to define or aspire to definitions based on global optima, for this would involve stating exactly what such optima would entail. This would require setting the problem up as selecting spaces and lines which were as 'fat' and as long as possible, respectively, with as small a number of spaces and lines as possible. This would require a definition of 'fat' which might be possible from the above measures such as compactness and/or convexity. It would also require formal optimization techniques which take the argument beyond the scope of this paper, but what space syntax requires are techniques to at least explore such optimization. These must await better definitions and explorations in the spirit of the current paper.

There are many issues which arise from this discussion. First, the algorithm does not guarantee that all axial lines will be connected. The isovist spaces selected (and hence the viewpoints) are of course connected, but the straight lines which approximate these may not be. It would be perfectly possible to construct a graph of relations between the chosen isovist spaces and to use this for subsequent relational analysis; this would be strongly connected by definition but it is unlikely that the links would follow straight lines. In fact, extending isovist analysis in a similar fashion is the basis of 'isovist integration analysis' proposed and implemented by Turner and Penn (1999). However, there is no reason why the axial map should be strongly connected if it is simply a summary of spatial orientations. The criterion imposed by Hillier and Hanson (1984) that the map be connected is an arbitrary one. It comes from wanting to imply directional, connective properties to the system that is subsequently used for analysis based on lines of sight which are assumed to be straight.

Second, it is possible to deal with overlapping areas and to deal with isovists which are tied at optimal values. In the longest line approach we could, for example, generate all isovists associated with the line, reduce the space accordingly from this mega-isovist, and continue in this fashion until all the space is covered. This would give rise to axial 
lines which were less dense and less connected than for the case where single isovists are identified at each pass of the method. In fact, we have implemented this in our applications to Gassin, and it is of interest to note that this is similar to one of the methods used by Peponis et al (1998) for generating axial lines which 'see everything' but do not necessarily get everywhere. A third problem relates to the appropriateness of the line for indicating space. In the ultimate axial map derived by this method, it is quite possible, indeed usual, to see two long lines almost in parallel spanning a space and ultimately intersecting. This is caused by one line being associated with the dominant isovist but that isovist not quite covering a portion of the space that generates its own axial line. At first sight, it might appear that the two lines are of equal dominance in that they are similar in length. However, one line is associated with the dominant isovist and, although the other line may be as long or longer, its isovist is less dominant than the first and thus has lesser importance in any subsequent analysis. In a sense, to read axial lines associated with this method, the relative importance of the isovist spaces must be considered, for there is a strict ranking of lines and spaces according to the criterion used in their selection.

The last issue here involves the meaning of the dominance ranking of axial lines and isovist spaces. In the case of the first variant in which isovists are selected according to the length of their line and then ties are broken according to the area covered, the ranking is based purely on length of line. In the case of the second more general variant, length of line is not the criterion as area or some other measure or attribute of the isovist is used for ranking. Lines are used only to summarize spaces and, if there is a correlation between the ranking of space and length of line, this is because area and length or compactness and length, etc, are related. It is easy to find criteria for selection and ranking which are not likely to be correlated. In fact, in many applications of space syntax there are very strong correlations between the length of axial lines and the subsequent accessibility values produced, for the simple reason that the longer the line the more likely it is to intersect with other lines. This is an issue that we will touch upon in our examples below but, once again, it represents another area of inquiry that is beyond the immediate concern of this paper.

\subsection{Comparing axial maps: the French town of Gassin}

The small town of Gassin was originally used by Hillier and Hanson (1984) to explain the rudiments of space syntax. It has subsequently been used as a test case by Peponis et al (1998) as well as by Jiang et al (2000) in an alternative reformulation of the theory. The example is manageable in that in the original application only 40 axial lines were defined, linking 139 'convex' spaces. In fact, the published data on the maps of the town differ enough to make the definition of axial lines and convex spaces ambiguous and this becomes critical in the methods that we use here which will identify every nook and cranny in the digital representation as being the potential origin of an isovist, and hence axial line. Nevertheless, the map that we have taken is sufficiently recognizable and intuitively appreciable as to make this a good test example.

We show the original plan, the convex spaces, and the axial map defined manually and intuitively by Hillier and Hanson (1984) in figures 5(a), (b), and (c) (over), respectively, in comparison with our own scanned map at the resolution used in the StarlogoT software in figure 5(d). All the subsequent measures in this section are based on this map which has dimensions of $196 \times 108$ pixels in the $x-y$ directions, with the space associated with the streetscape between the buildings being 8129 (square) pixels in area. The longest diagonal in this plan is some 214 units of distance and this provides a benchmark to all subsequent calculations and results reported here. All distance and area measures are rounded to integers and ratios and related statistics are given to three 


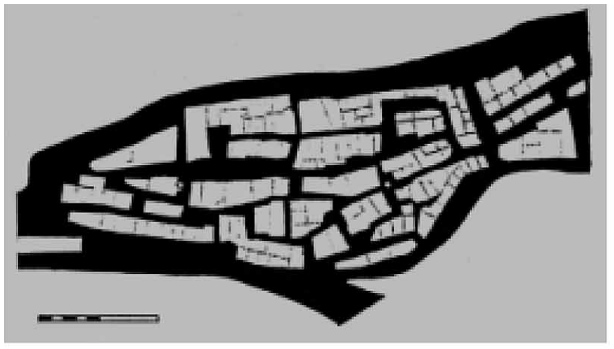

(a)

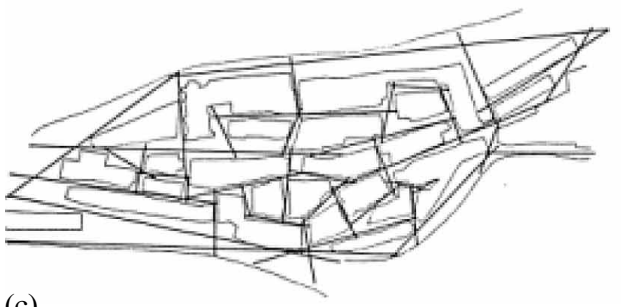

(c)

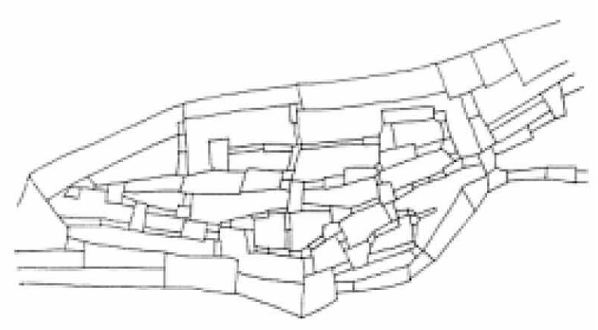

(b)

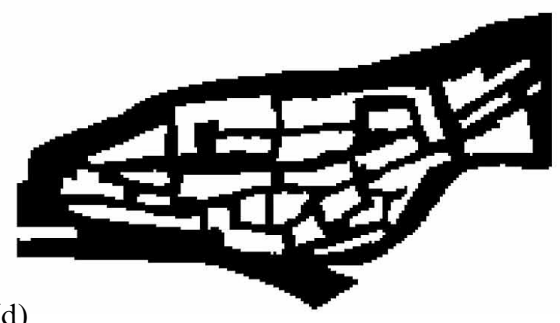

(d)

Figure 5. Basic data for the town of Gassin: (a) building blocks and street parcels; (b) partition into convex sets; (c) the original axial map; (d) the digitized map.

decimal places. In the analysis that follows, we will also measure different areas of the isovists associated with axial lines so that we can make comparisons between different variants of our own algorithm. Unfortunately it is not possible to associate axial lines in the original example with the convex spaces that they summarize as there is no unique mapping from lines to spaces and thus all comparisons between the results generated here, the original, and the examples developed by Peponis et al (1998) will solely be in terms of numbers and lengths of axial lines.

We will first generate a series of isovist fields for Gassin by using agent-based methods which walk an agent to all points in the viewshed associated with a given viewpoint. This method generates isovists and isovist fields which are then used to associate different geometric and related measures of properties of viewsheds at each point in the space (Batty, 2001; Batty and Jiang, 2000). Agents walk $180^{\circ}$ in increments of $1^{\circ}$ forwards and backwards from each viewpoint, measuring a series of geometric characteristics from which the set of relevant measures identified earlier in equations (1) - (6) are thence computed. The program for Gassin currently takes about 21 minutes on a MacIntosh i-Book (with PowerPC G3 processor running at $366 \mathrm{MHz}$ ). In the analysis we use nine measures, four of which involve the distances $d_{i}^{\min }, d_{i}^{\max }, \bar{d}_{i}$, and $\Delta_{i}^{\max }$, the area and perimeter measures $a_{i}$ and $p_{i}$, and three of the ratio measurescompactness, convexity, and circularity, $\Gamma_{i}, \Psi_{i}$, and $\Theta_{i}$. We have not used the minimum diametric length or the drift parameter, nor all the means and variances shown earlier, as there is a surfeit of possibilities which all need to be explored in another context. In our ArcView software, a full set of measures is computed (see http://www.casa.ucl.ac.uk/ sanjay/software_isovistanalyst.htm).

The first stage in generating axial lines is to compute the various isovist fields which are then used for ordering the spaces for which axial lines are used as a summary. The nine fields based on each given measure are shown in figure 6 where the mean and standard deviation of each measure are also shown to provide some comparative basis for the statistics used below. The minimum distance field is the 


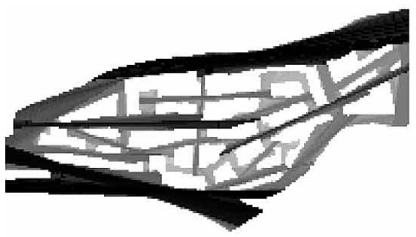

(a) Maximum diametric length $\left\{\Delta_{i}^{\max }\right\}, \mu=87, \sigma=36$

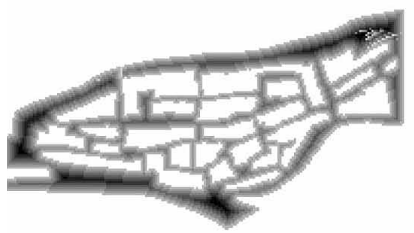

(d) Minimum distance $\left\{d_{i}^{\min }\right\}, \mu=2.538, \sigma=1.606$

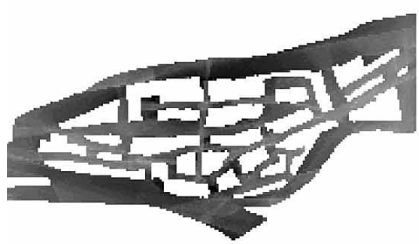

(g) Convexity

$\left\{\Gamma_{i}\right\}, \mu=0.254, \sigma=0.040$

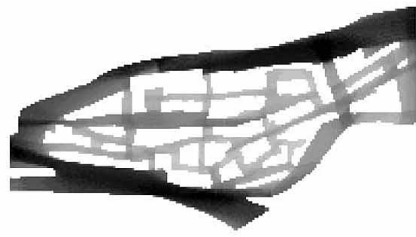

(b) Area

$\left\{a_{i}\right\}, \mu=869, \sigma=528$

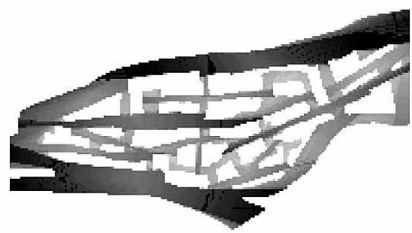

(e) Farthest distance $\left\{d_{i}^{\max }\right\}, \mu=72, \sigma=33$

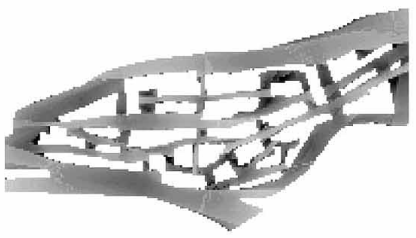

(h) Compactness

$\left\{\Psi_{i}^{\max }\right\}, \mu=0.338, \sigma=0.123$

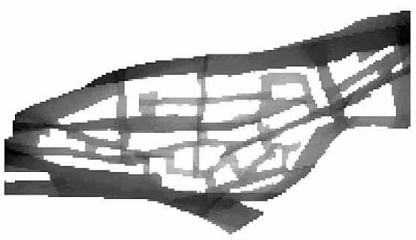

(c) Perimeter

$\left\{p_{i}\right\}, \mu=390, \sigma=124$

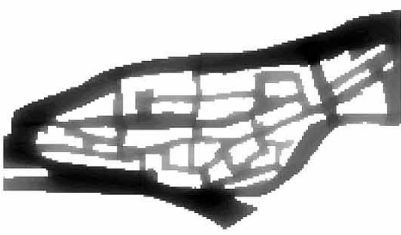

(f) Average distance

$\left\{\bar{d}_{i}\right\}, \mu=22, \sigma=7.566$

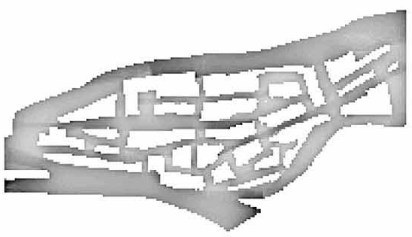

(i) Circularity

$\left\{\Theta_{i}\right\}, \mu=0.271, \sigma=0.071$

Figure 6. Isovist fields for nine standard geometric measures.

easiest to explain in that it depends entirely on how near the observer is to some edge, and the field is largely structured by the width of each street. The largest distances and diameters are highly correlated $\left(r^{2} \approx 0.835\right)$, with the axial structure of the system clearly marked in these fields. In fact, although we will not do so, these measures can be used directly in the extraction of axial lines although this would depend not on geometric issues which drive the current quest but on image-processing techniques (Ratti, 2002). We have begun this process and first results are reported in Carvalho and Batty (2003). Average distance smoothes the kinds of striations which characterize fields based on minimum and maximum distances but, in all these cases, the measures show the existence of visual threshold particularly at points of discontinuity where vistas close or open up.

Area and perimeter are highly correlated at $r^{2} \approx 0.736$, but it is area and average distance that have the strongest correlation with $r^{2} \approx 0.866$. Area is correlated with the maximum diametric distance as well, which is important as these two measures are central to the algorithm to be used in extracting axial lines which cover isovist areas in the most efficient way. The three ratio coefficients that measure how close each isovist is to circles, lines, and related geometric figures have rather low correlations with all the other measures, including each other. This is largely because these measures detect very different features of the system. Convex spaces are the exception rather than the rule in street systems, such as the ones we deal with here, the same being true of compact and circular spaces. What these fields show is that smaller spaces in this system tend to be more compact and convex, although the irregularity of the system even in large 
wide streets tends to destroy any meaningful association with direct areal or distance measures. Convexity is highest in the widest streets, compactness in the smaller shorter streets, and circularity at the edges of the system as an artifact of the measure itself. As we might expect, there is little association with the pattern of isovists which tend to be nonconvex, noncompact, and noncircular.

These fields form the measures from which axial lines can be extracted by using the algorithm presented earlier and illustrated in figure 4 . In essence, the procedure works with a specific measure for each viewshed or isovist, ordering the isovists according to this measure, usually from largest to smallest, starting with the largest and selecting the isovist associated with this as being the most important in the system. This space is then approximated by the maximum diametric distance $\Delta_{l}^{\max }$, the overall space reduced by this isovist, and then the next viewpoint and isovist associated with the next largest measure in the remaining space selected. The procedure continues in this way, approximating each subsequent space by its relevant maximum diameter until all space has been covered. We have applied six variants of this algorithm with different measures in each case. The first baseline case adopts the maximum diameter as the measure to optimize and thus the procedure selects space associated with this maximum which is then approximated by the same diameter. The second method is based on area, the third on average distance, and the remaining three use the convexity, compactness, and circularity coefficients for the dominance ranking.

We show the results of these applications in figures 7(a) and (b). In figure 7(a), for the first application based on selection according to the longest diametric length of each isovist, we show four related maps: the isovists associated with the ranking with most important as the top layer and all others in order beneath the first; the number of overlaps of each isovist which gives an impression of how central different locations are within the space; the viewpoints of each isovist; and their axial lines - the maximum diametric diameters which form the axial map. In fact, it is essential to read these results in terms both of isovist spaces and of axial lines because the relative importance is based first and foremost on the spaces, not on the lines used to approximate them. There is an immediate connection between spaces and lines, one that does not formally exist in space syntax, despite the loose association between axial lines and convex spaces. It is tempting to read the axial lines separately in the manner of traditional space syntax where the underlying space is almost forgotten, but the line is only one side of the coin in interpreting the importance of different spaces making up a morphology. Notwithstanding the characteristic used to rank importance, the length of the axial line and the area covered by all the associated isovists are basic measures which indicate the efficiency of the application. We illustrate only the axial maps for each of the remaining five applications in figure $7(\mathrm{~b})$.

It is immediately clear from these results that the rankings based on longest lines, largest areas, and largest average distances give results that are much more efficient than those which depend on the geometric ratios which do not really reflect the linearity of the underlying street system. We illustrate a series of quantitative measures relating to the number and length of lines and areas of associated isovist spaces for each of the six applications in table 1 (over), where we also contrast these with the more minimal information we have for the Hillier and Hanson (1984) and Peponis et al (1998) applications. We need to be clear about what is shown here. We will now define the total number of isovists and axial lines generated from each application by $L$, the area of the selected isovist by $a_{l}(l=1,2, \ldots, L)$, and the length of the maximum diametric distance by $\Delta_{l}^{\max }(l=1,2, \ldots, L)$. In table 1 we show the number $L$, the total line length $\sum_{l} \Delta_{l}^{\max }$, the average line length $\sum_{l} \Delta_{l}^{\max } / L$, the total area $\sum_{l} a_{l}$, and the average area $\sum_{l} a_{l} / L$. As the selected isovists overlap, we can compare the total area 


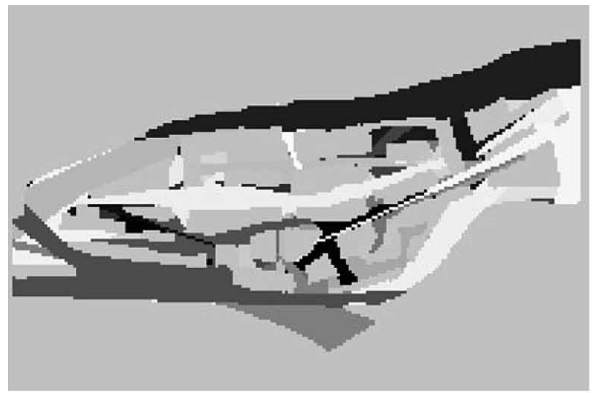

Isovists in dominance order

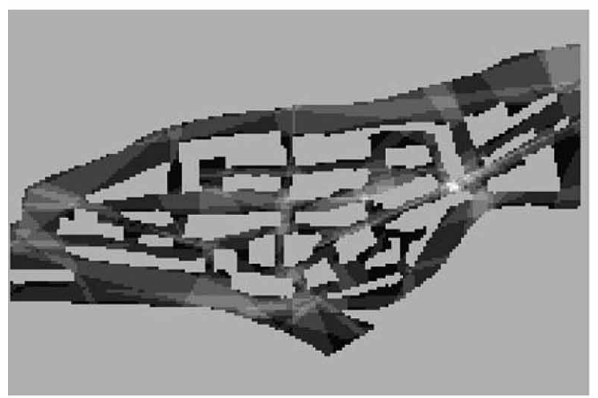

Overlap count of dominant isovists

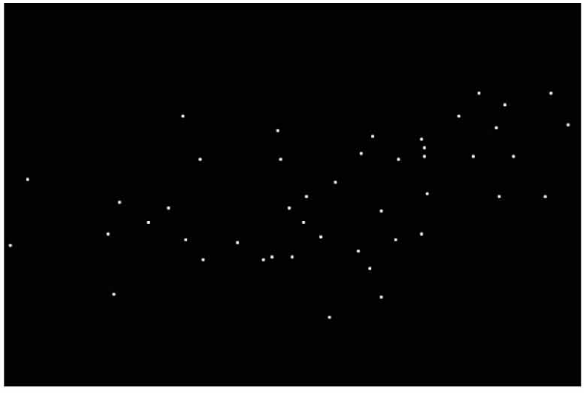

Viewpoints of dominant isovists

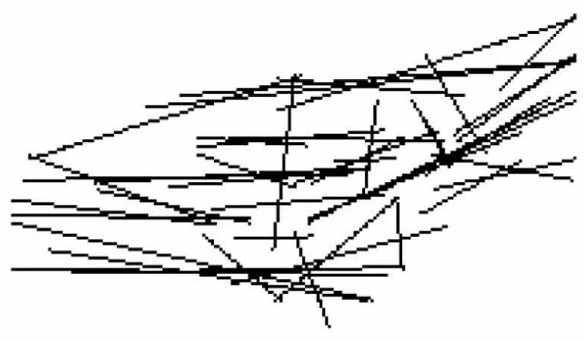

Axial lines as maximum diameters

(a)

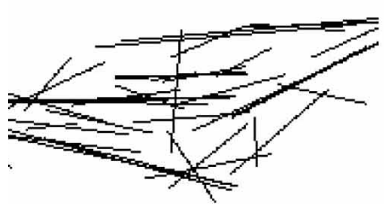

Area

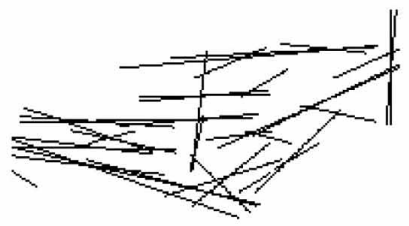

Average distance

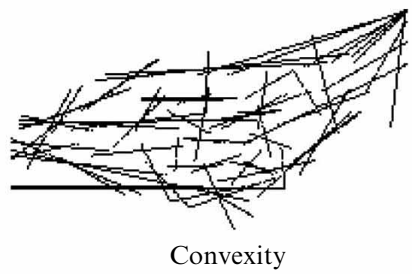

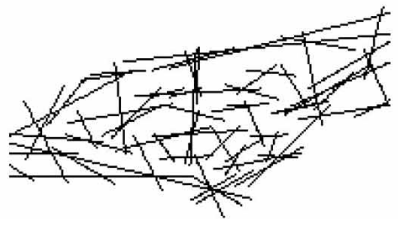

Compactness

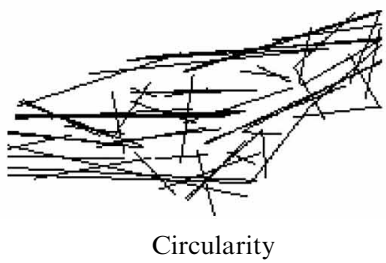

(b)

Figure 7. Axial lines generated by the isovist-sorting algorithm: (a) isovists, overlaps, viewsheds, and axial maps for the longest line sort; (b) axial maps for the five remaining sorts.

with the actual area of the streetscape (which in this case is 8129 units of area). We thus form the ratio $\sum_{l} a_{l} / 8129$ which gives the relative duplication of space from such overlaps in comparison with a system where there is no such duplication, as, for example, in a system divided into mutually exclusive convex spaces as in figure 5(b).

As we implied above, formal optimization procedures have never been developed within space syntax in the quest to generate lines that best summarize convex spaces, and thus there is no criterion by which to judge the appropriateness of an axial map. We would argue, however, that this is essential if we are to make progress. To this end, 
Table 1. A comparison of methods for generating axial lines.

\begin{tabular}{|c|c|c|c|c|c|c|c|}
\hline Method & $\begin{array}{l}\text { Number } \\
\text { of axial } \\
\text { lines } L\end{array}$ & $\begin{array}{l}\text { Total } \\
\text { line } \\
\text { length } \\
\sum_{l} \Delta_{l}^{\max }\end{array}$ & $\begin{array}{l}\text { Average } \\
\text { line } \\
\text { length } \\
\sum_{l} \Delta_{l}^{\max } / L\end{array}$ & $\begin{array}{l}\text { Total } \\
\text { area } \\
\text { covered } \\
\sum_{l} a_{l}\end{array}$ & $\begin{array}{l}\text { Average } \\
\text { area } \\
\sum_{l} a_{l} / L\end{array}$ & $\begin{array}{l}\text { Area } \\
\text { covered to } \\
\text { total area } \\
\sum_{l} a_{l} / 8129\end{array}$ & $\begin{array}{l}\text { Efficiency } \\
\text { ratio } \\
\Xi\end{array}$ \\
\hline $\begin{array}{l}\text { Hillier and } \\
\text { Hanson }\end{array}$ & 40 & 1565 & 38 & $\mathrm{nr}$ & $\mathrm{nr}$ & $\mathrm{nr}$ & $\mathrm{nr}$ \\
\hline Peponis et al 1 & 13 & 959 & 74 & $\mathrm{nr}$ & $\mathrm{nr}$ & $\mathrm{nr}$ & $\mathrm{nr}$ \\
\hline Peponis et al 2 & 37 & 1635 & 44 & $\mathrm{nr}$ & $\mathrm{nr}$ & $\mathrm{nr}$ & $\mathrm{nr}$ \\
\hline Longest line & 46 & 3211 & 70 & 21500 & 467 & 2.645 & 308 \\
\hline Largest area & 39 & 2592 & 66 & 23225 & 596 & 2.857 & 349 \\
\hline $\begin{array}{l}\text { Largest average } \\
\text { distance }\end{array}$ & 36 & 2276 & 63 & 20628 & 573 & 2.538 & 326 \\
\hline $\begin{array}{l}\text { Greatest } \\
\text { convexity }\end{array}$ & 72 & 3409 & 47 & 29971 & 416 & 3.687 & 633 \\
\hline Most compact & 67 & 2637 & 39 & 21929 & 327 & 2.698 & 557 \\
\hline Nearest circular & 60 & 3768 & 63 & 26281 & 438 & 3.234 & 418 \\
\hline
\end{tabular}

we suggest that a critical measure is the area associated with a unit line for any system of isovist areas and axial lines. We wish to minimize this average to find a set of lines which covers the area in the most parsimonious way. We also want to minimize the number of these lines as well as their areal linearity. Accordingly, we define the measure

$$
\Xi=L\left(\frac{\sum_{l} a_{l}}{\sum_{l} \Delta_{l}^{\max }}\right) .
$$

which we will use as a test of efficiency. We show this in table 1 and there it is immediately clear that the distance and area methods come out best. The method which optimizes the selection of isovists based on the length of their maximum diameter generates 46 lines which have an average length of 70 units covering a unit area of 21500 . This contrasts with the second method in which isovists are selected on the basis of their area; these yield 39 lines but these on average are shorter at 66 and more area is covered at 23225 unit area. The method in which average distance is optimized yields even less lines at 36 , but the line is shorter at 63 and the area covered smaller at 20628 . The ratio methods all generate much larger numbers of lines, with the convexity measure generating twice as many (72) lines as the average distance. The efficiency ratio $\Xi$ bears all this out, with the efficiency ranking from the longest line method (best), largest average distance, largest area, greatest circularity, compactness, and convexity (worst). The key issue here is that the number of axial lines is not in and of itself the most important criterion, for this must be matched against their length and the space that they summarize.

\subsection{The statistics of axial lines: a preliminary analysis}

To conclude our analysis of these six applications we will make a brief foray into the statistical form of the isovist fields and the lines that are generated from the sorting procedures used to partition them into significant viewpoints. An attempt was made by Batty (2001) to initiate such analysis for a range of parameters describing such fields, but here we concentrate exclusively on the maximum diametric distance associated with these spaces. There is little doubt that this area is yet another in space syntax analysis which has never been researched and is an essential focus in refining and extending the theory. The distribution $\left\{\Delta_{i}^{\max }\right\}$ over all 8129 isovists for Gassin is 
nonnormal in that its frequency distribution is bimodal which is a characteristic of linear distances in isovist fields for street systems noted in earlier applications (Batty, 2001). The bimodality essentially classifies isovists into long and short vistas, which would appear to be consistent with systems which are dominated by a strict hierarchy of streets. The frequency distribution of lengths, however, is not as useful a plot as the cumulative frequency, and the form that we prefer here, much used in scaling analysis, is called the rank-size. This is based on a plot of the distance lengths against their rank which we show for the set $\left\{\Delta_{i}^{\max }\right\}$ in figure 8 where we show the frequency, cumulative frequency, and the rank-size, which is a reverse plot of the cumulative frequency from largest to smallest distance.

The rank-size is essentially linear with $r^{2} \approx 0.983$. There is thus no evidence of scaling in the distribution of isovist lengths within their isovist field. However, in any process of selection which begins with the largest lengths and orders these so that the space under consideration is successively reduced, it is likely that scaling will be
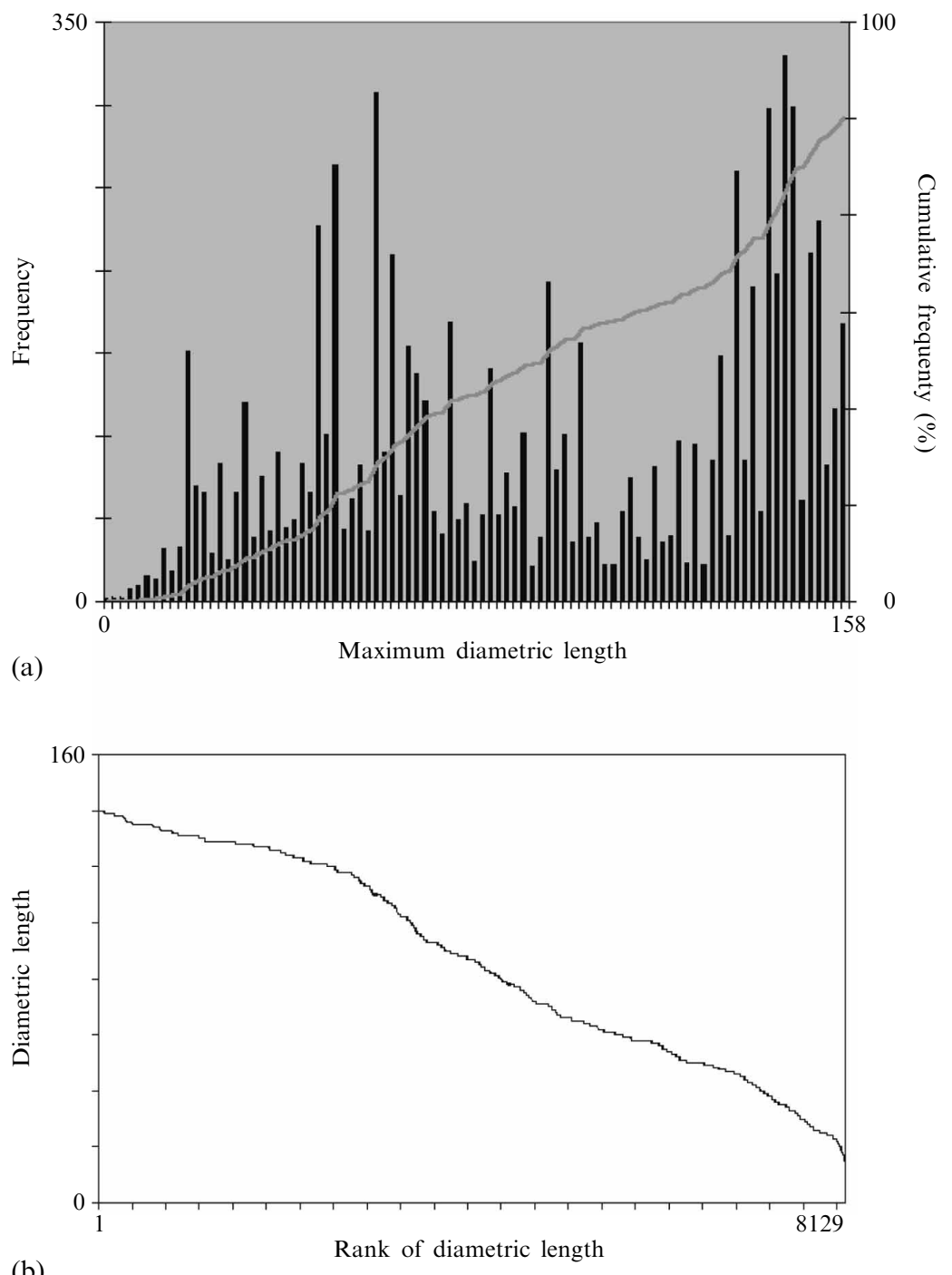

(b)

Figure 8. Frequencies and rank-size distributions of maximum diametric lengths. 
introduced into this process. In short, the largest isovist in the system is first identified and as this is likely to intersect with many of the other largest isovists, then most of the largest lengths get ruled out of consideration at this first stage. As the process continues, the spaces get smaller and the isovists at that length size become increasingly less likely to intersect one another and thus more and more smaller isovist lengths are included. In short, the algorithm we use to select isovist lengths introduces scaling into a system that does not have scaling already. This is because we assume that there is a strict order of space from largest to smallest and that the number of large spaces is likely to be considerably smaller than the number of small spaces. This is the criterion for scaling which we might expect when we examine the distribution of isovist lengths which form the axial map.

In figure 9 we have graphed the rank - size relations based on each set $\left\{\Delta_{l}^{\max }\right\}$ for each of the six applications of the algorithm. These show a degree of scaling although, when presented in logarithm form, they imply something closer to log normality than the classic Pareto power function. In table 2 we show these relations fitted for two functions: the traditional power-law form $\left(\Delta_{l}^{\max }\right)^{\prime}=\alpha r_{l}^{-\beta}$ and the exponential form $\left(\Delta_{l}^{\max }\right)^{\prime}=\alpha \exp \left(-\beta r_{i}\right)$, where the results are all significant for both models, with the coefficients of determination greater for the exponential than the power laws. We also compare the line lengths for the Hillier and Hanson (1984) and Peponis et al (1998) examples in this table where it is also clear that the axial lines produced by these traditional methods are also scaling. This is good initial evidence that axial lines are scaling because of the process of their selection and the general space syntax assumption that it is essential to identify the importance of spaces according to their area, with the largest spaces taking priority.

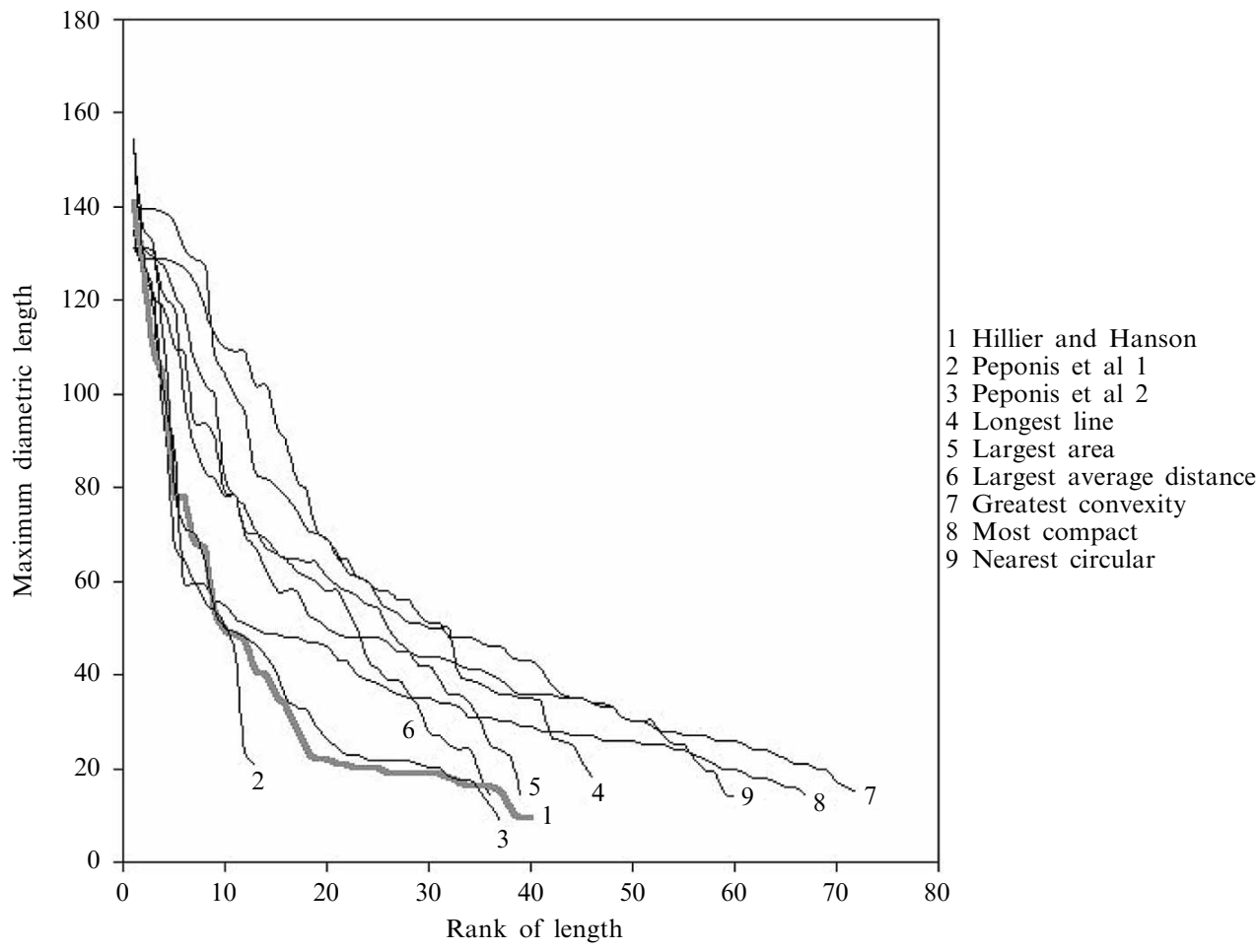

Figure 9. Rank - size distribution of maximum diametric lengths. 
Table 2. Estimation of power and exponential relations for axial distances.

\begin{tabular}{|c|c|c|c|c|c|c|c|}
\hline \multirow[t]{2}{*}{ Method } & \multirow{2}{*}{$\begin{array}{l}\text { Number } \\
\text { of axial } \\
\text { lines, } L\end{array}$} & \multicolumn{3}{|c|}{$\begin{array}{l}\text { Power } \\
\left(\Delta_{l}^{\max }\right)^{\prime}=\alpha r_{l}^{-\beta}\end{array}$} & \multicolumn{3}{|c|}{$\begin{array}{l}\text { Exponential } \\
\left(\Delta_{l}^{\max }\right)^{\prime}=\alpha \exp \left(-\beta r_{i}\right)\end{array}$} \\
\hline & & $\alpha$ & $\beta$ & $r^{2}$ & $\alpha$ & $\beta$ & $r^{2}$ \\
\hline Hillier and Hanson & 40 & 2.488 & -0.563 & 0.798 & 1.995 & -0.025 & 0.914 \\
\hline Peponis et al 1 & 13 & 2.409 & -0.542 & 0.761 & 2.262 & -0.066 & 0.939 \\
\hline Peponis et al 2 & 37 & 2.414 & -0.587 & 0.766 & 2.048 & -0.027 & 0.934 \\
\hline Longest line & 46 & 2.403 & -0.548 & 0.913 & 2.200 & -0.018 & 0.979 \\
\hline Largest area & 39 & 2.273 & -0.521 & 0.941 & 2.166 & -0.020 & 0.944 \\
\hline $\begin{array}{l}\text { Largest average } \\
\text { distance }\end{array}$ & 36 & 2.528 & -0.587 & 0.789 & 2.169 & -0.023 & 0.958 \\
\hline Greatest convexity & 72 & 2.422 & -0.792 & 0.931 & 1.997 & -0.011 & 0.944 \\
\hline Most compact & 67 & 2.344 & -0.724 & 0.817 & 1.889 & -0.010 & 0.907 \\
\hline Nearest circular & 60 & 2.438 & -0.775 & 0.929 & 2.172 & -0.015 & 0.976 \\
\hline
\end{tabular}

\section{An improved algorithm: the full Gassin application}

\subsection{Scale and resolution in space syntax}

It is very clear from the discussion so far that the scale at which any spatial system is represented has an important effect on the way the degree of detail is represented. This in turn is likely to affect the number of spaces into which it is partitioned, the best example being a building or streetscape in which there are thin objects (such as columns) not judged to be part of the building fabric. At a certain level of resolution, these objects will disappear as the scale becomes too coarse for their detection. The same would be true for detailed crenellations, entries, and such like in the building fabric. Consider the plan of Gassin in figure 5. At the level of resolution at which it is represented by Hillier and Hanson (1984) in figure 5(a) or the slightly lower level of resolution used in its digitization in figure 5(d), details of less than $2 \mathrm{~m}^{2}$ would disappear and thus many nooks and crannies essential to the visualscape would be lost. This line of argument immediately leads to the notion that the number of convex spaces or distinct viewpoints used to form isovists will vary with the level of resolution. In turn this means that the number of axial lines derived traditionally by manual means or by using the algorithm developed here would vary. As more detail is picked up at ever finer scales, the number of axial lines increases.

Analysis of scale and aggregation is central to contemporary spatial analysis. The most comprehensive statement is by Openshaw (1984) who identified crucial changes posed by aggregating scale as the modifiable areal unit problem. In essence, he argued that, as the scale of representation changes and if the morphology of the space which is used to classify spatial variation changes too, conventional spatial analysis would yield differing results which, in the extreme, might lead to contradictory inferences at different scales. A variant of this problem in terms of measurement involves the notion of the fractal line in that, as the scale becomes finer, more and more detail is picked up, leading to changes in standard measurements, such as the length of a line. This has been demonstrated many times; the most famous examples being for coastlines (Mandelbrot, 1967) and political borders (Richardson, 1961).

We can easily demonstrate this for Gassin using our trial software which is structured so that the level of resolution (number of pixels used to detect the streetscape and building outlines) is easily varied. The applications in the previous sections are based on a $2 \times 2$ pixel size which generates a $201 \times 201$ pixel map within which the streetscape occupies 8129 pixels. We can vary this to a $4 \times 4$ pixel size with a $101 \times 101$ map, an $8 \times 8$ pixel size which gives a $51 \times 51$ map, and a $16 \times 16$ pixels size giving a 
$25 \times 25$ map. When we represent Gassin at these different levels, the number of isovist fields, sorted by maximum diametric length (and hence axial lines) reduces consistently and dramatically as we show in table 3. As we have only four levels of aggregation, we can only speculate as to the nature of the relationship between the number of axial lines and the level of resolution but, as resolution is a two-dimensional variable and the axial line one-dimensional, we might expect this relation to be exponential. Indeed from table 3 it is clear that, as the number of pixels associated with the streetscape increases, the number of axial lines increases but at an exponentially decreasing rate.

Table 3. Axial lines for Gassin at different scales.

\begin{tabular}{lllll}
\hline $\begin{array}{l}\text { Level of pixel } \\
\text { resolution }\end{array}$ & $\begin{array}{l}\text { Size of } \\
\text { pixel space }\end{array}$ & $\begin{array}{l}\text { Pixels in } \\
\text { streetscape }\end{array}$ & $\begin{array}{l}\text { Number of } \\
\text { axial lines }\end{array}$ & $\begin{array}{l}\text { Time for sorting } \\
\text { algorithm (minutes) }\end{array}$ \\
\hline $2 \times 2$ & $201 \times 201$ & 8129 & 46 & 21 \\
$4 \times 4$ & $101 \times 101$ & 2329 & 26 & 4 \\
$8 \times 8$ & $51 \times 51$ & 737 & 7 & 2 \\
$16 \times 16$ & $25 \times 25$ & 204 & 2 & 1.5 \\
\hline
\end{tabular}

\subsection{Axial lines and isovist fields in GIS: the ArcView extension}

To demonstrate the importance of this question of scale, we have implemented the algorithm shown in figure 4 as an extension to the desktop GIS ArcView which enables us to import any vector-based image of the building outline into the GIS. In fact, we first convert the raster scan of Gassin from Hillier and Hanson's (1984) book into the appropriate vector map using the freeware WinTopo. We then set a grid of viewpoints at any level of resolution (akin to the pixel raster of StarlogoT), fix any angle of

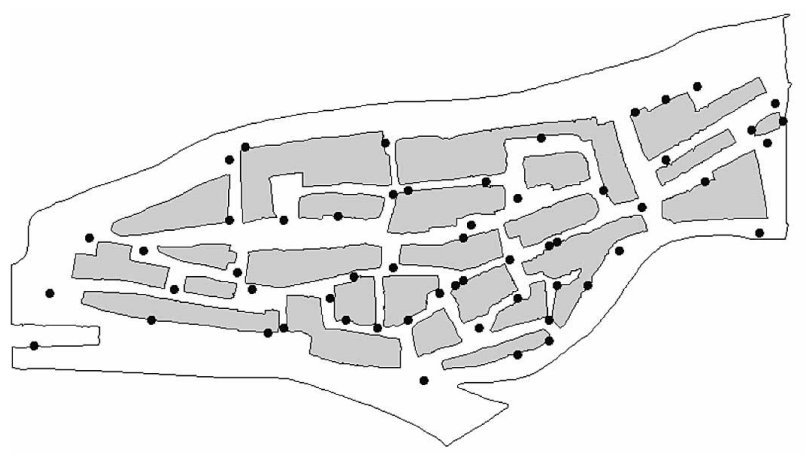

(a)

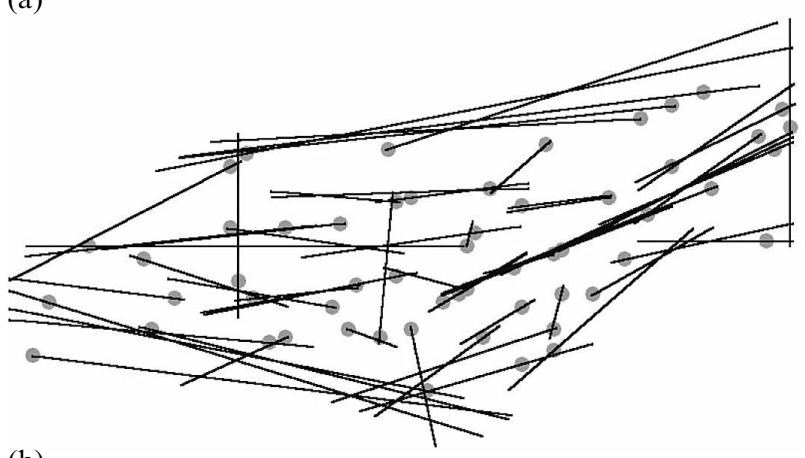

(b)

Figure 10. Isovist centroids and axial lines computed from the ArcView extension. 
incremental rotation or sweep around the viewshed, and compute isovist polygons from intersections of the rays from the viewpoint to the building outlines. As the level of resolution of the building outlines is invariant to the grid of viewpoints, then the isovist polygons will also be relatively invariant to the resolution of this grid. As the grid gets denser, the isovist polygons will change in shape but, as they are based on intersections of the rays with a fixed outline, this variation will be considerably smaller than that posed by approximating both viewpoints and building outlines by a standard grid as we do in StarlogoT. The greatest variation in the shape of isovists will thus come from differences in the angle of rotation, not the density of the grid itself. The major advantage of implementing this in ArcView is the fact that the spatial system can be represented in vector form, which is stable regardless of the number and resolution of the isovists themselves.

We compute and sort the isovists based on the strict hierarchy of maximum diametric lengths using the detailed plan of Gassin shown in figure 10(a). We have set the parameters - the number of viewpoints, and the incremental angle - at the same levels of resolution as the applications given in the previous section, with around 8000 viewpoints and a $1^{\circ}$ angle of sweep. However, as figure 10(a) shows, the town plan is at a much higher level of resolution than the previous applications and thus the number of irregular building faces far exceeds those of the digitized plan in figure 5(d). Thus one would expect there to be more isovists generated through the sorting procedure as more detail is being picked up. This is borne out in the fact that the number of isovists (and hence axial lines), selected is 56, some $20 \%$ more than the cruder digitization but consistent with the relation implied in table 3 . These are shown in

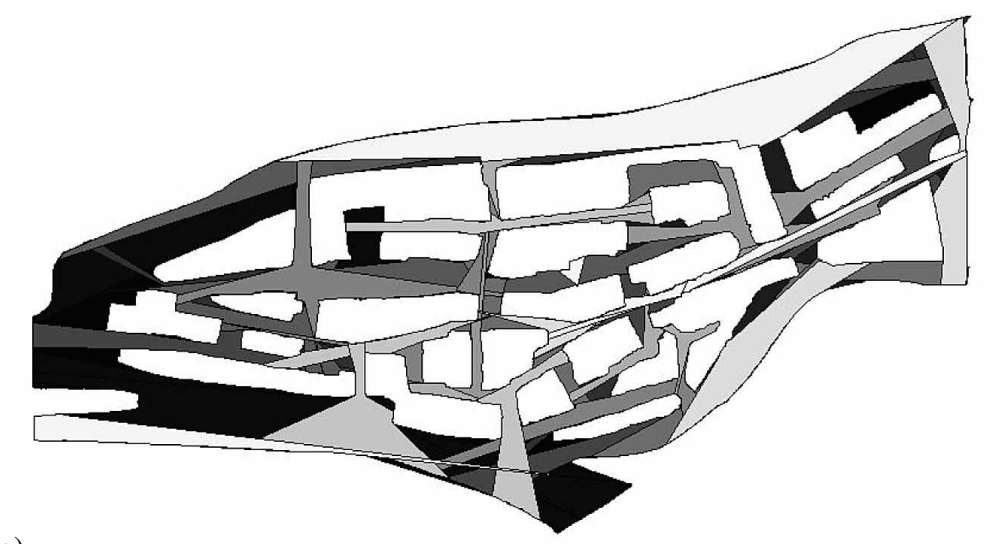

(a)

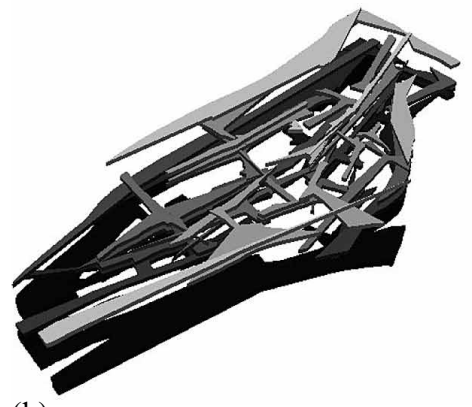

(b)

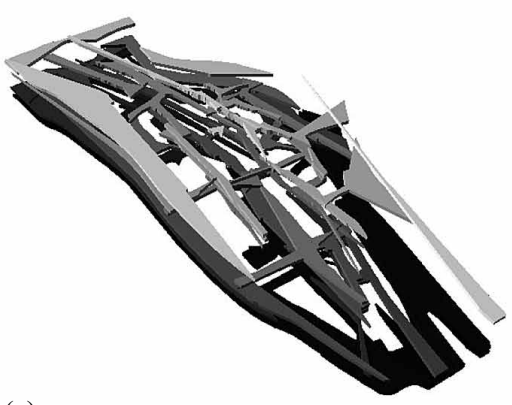

(c)

Figure 11. Two-dimensional and three-dimensional views of the dominance hierarchy of overlapping isovists. 
figure 10(b). However, what this application suggests is that the number of axial lines would vary much less when the density of viewpoints changes than in the case where the level of resolution of the building outlines and streetscape change.

There are many advantages to implementing such algorithms within well-developed standard software such as ArcView which is extremely modest in cost. In particular, the many extensions that can be used to visualize and compute spatial metrics for maps and layouts help extend the analysis. What we are able to do here is to visualize the way isovists overlap with one another much more easily than we did in figure 7 , by invoking the 3d-Analyst extension. In figure 11 we show two perspective views of the overlap where we have colored the isovists according to the scale of their dominance and ordered them in three dimensions from top to bottom. This shows immediately how axial lines are a very weak way of visualizing this kind of spatial complexity. It also shows that the sorting algorithm we use always leads to isovists which are connected through their overlaps because the original streetscape space is connected.

\section{Conclusions: next steps}

One immediate objection to the methods we have developed here is that they do not solve the traditional space syntax problem as originally formulated by Hillier and Hanson (1984). We sidestep the problem by replacing the task of generating a connected axial map which spans a partition of space into convex sets with one in which we generate a map, not necessarily connected, which spans a partition of the space into connected viewsheds, sorted with respect to length or area, or any other criterion associated with the viewsheds. In this sense, we might be accused of adopting a quick fix to the problem. We would agree with this and consider that the space syntax problem needs to be entirely reformulated with each of its assumptions (about the need to partition into convex spaces, approximate these by straight lines, and ensure that these lines connect) all coming under scrutiny in terms of the best way of representing urban morphology. Moreover, this representation must be tied much more strongly to behavioral issues, to the nature of economic activities in cities which is the core of urban geography, and to ways in which people interact through various modes of transport.

Space syntax needs to be considered as one version of the generic problem of spatial representation which involves simplification of geometric form to reflect more parsimonious ways of understanding the importance of different spaces and the way they are related. In this sense, the axial line may not be the appropriate unit of analysis but something more basic such as the parcel or even some fine-level grid should be explored. More than one axial line might be used to approximate an isovist as one anonymous referee of this paper suggested and such a wealth of possibilities are all worthy of testing. In short, space syntax needs to embrace and relate to other approaches to urban morphology, such as shape grammars, $Q$-analysis, cellular systems, fractal representations, and so on. This is the wider and longer term agenda. In the shorter term, the strictures posed in summarizing space by straight lines need to be explored further, and this in turn raises questions as to the purpose of defining such lines when simpler and more obvious ways or relating the spaces that they summarize are readily available.

With respect to the actual methods presented here, there is much work to do. The basic algorithm we have developed sorts isovists according to a very strict dominance ranking. We need to relax this in the manner that we noted in our final example where the larger isovist envelope based on the longest axial line was constructed and then used as a basis for ranking. We also intend to explore ways in which isovists might be used as seeds in some evolutionary solution to generating spatial subdivisions which 
meet a variety of criteria, thus synthesizing bottom-up criteria with top-down. This will lead us to pose the partition problem in a rather different way, taking us to global rather than local optimization.

There are other improvements to the algorithms developed here that we might make rather quickly. So far, space syntax has not been able to handle the third dimension, largely because it remains a manual method in terms of its representation through axial lines. However, it is easy to build terrain into the raster-based viewshed representations which have been adopted here and, although our examples do not deal with varying terrain (because the terrain of Gassin was not published in the original application), it would be a simple matter to add height to the raster and to truncate the ray tracing when distant areas disappear from sight. True extensions to deal with threedimensional environments are on the horizon but, once again, real progress can be made only if the basic space syntax problem is reformulated.

In short, a major research program is required which must be part of our wider quest to develop better ways of representing urban morphology so we can understand the ways building and townscapes evolve through organic growth and change as well as through design. We are already examining a whole series of extensions to the problem of isovist representation and sorting using the methods that we have presented here while we are also working on ways of representing relations between spaces by using standard ideas of graph theory which are in use in other areas. These, we hope, will provide us with finer foundations for space syntax in particular, and the study of urban and architectural morphology in general.

\section{References}

Atkin R H, 1974 Mathematical Structure in Human Affairs (Heinemann Educational Books, London) Batty M, 2001, "Exploring isovist fields: space and shape in architectural and urban morphology" Environment and Planning B: Planning and Design $28123-150$

Batty M, Jiang B, 2000, "Multi-agent simulation: computational space-time dynamics in GIS", in Innovations in GIS VII: GIS and Geocomputation Eds P Atkinson, D Martin (Taylor and Francis, London) pp $55-71$

Batty M, Tinkler K J, 1979, "Symmetric structure in spatial and social processes" Environment and Planning B $63-27$

Benedikt M L, 1979, “To take hold of space: isovists and isovist fields" Environment and Planning $B$ $647-65$

Blum H, Nagel R, 1978, "Shape description using weighted symmetric axis features" Pattern Recognition $10167-180$

Carvalho R, Batty M, 2003, "A rigorous definition of axial lines: ridges on isovist fields", WP 69, Centre for Advanced Spatial Analysis, University College London, http://www.casa.ucl.ac.uk/ working_papers/paper69.pdf

Dalton R C, Dalton N, 2001, "OmniVista: an application of isovist field and path analysis", in Proceedings, Third International Symposium on Space Syntax, Atlanta 200125.1 -25.10,

The Bartlett Graduate School, University College London

Desyllas J, Duxbury E, 2001, "Axial maps and visibility analysis: a comparison of their methodology and use in models of urban pedestrian movement", in Proceedings, Third International Symposium on Space Syntax, Atlanta $200127.1-27.13$

Hillier B, 1996 Space is the Machine: A Configurational Theory of Architecture (Cambridge University Press, Cambridge)

Hillier B, Hanson J, 1984 The Social Logic of Space (Cambridge University Press, Cambridge)

Intelligent Space, 2002 FATHOM: Visibility Graph Analysis Software Intelligent Space Partnership, 68 Great Eastern Street, London EC2A 3JT, http://www.intelligentspace.com/tech/fathom.htm

Jiang B, Claramunt C, Karlqvist B, 2000, "An integration of space syntax into GIS for modeling urban spaces" International Journal of Applied Earth Observation and Geoinformation (JAG) $2161-171$

Llobera M, 1996, "Exploring the topography of mind: GIS, social space and archaeology" Antiquity $70612-622$ 
Mandelbrot B B, 1967, "How long is the coast of Britain? Statistical self similarity and fractal dimension" Science 155 636-638

Openshaw S, 1984 The Modifiable Areal Unit Problem Concepts and Techniques in Modern Geography 38 (Geobooks, Norwich)

O'Rourke J, 1987 Art Gallery Theorems and Algorithms (Oxford University Press, New York)

Penn A, Conroy R, Dalton N, Dekker L, Mottram C, Turner A, 1997, "Intelligent architecture: new tools for three dimensional analysis of space and built form" Proceedings, First International Symposium on Space Syntax, London 1997 Space Syntax Laboratory, University College London, London, $30.1-30.19$

Peponis J, Wineman J, Rashid M, Kim S H, Bafna S, 1997, "On the description of shape and spatial configuration inside buildings: convex partitions and their local properties" Environment and Planning B: Planning and Design 24761 - 781

Peponis J, Wineman J, Bafna S, Rashid M, Kim S H, 1998, “On the generation of linear representations of spatial configuration" Environment and Planning B: Planning and Design $25559-576$

Rana S, 2002, "Fast Viewshed computation using topographic features", in Proceedings, GISRUK 2002, Sheffield pp 16-21, http://www.shef.ac.uk/gisruk/

Rana S, Morley J, 2002, "Surface networks", WP 43, Centre for Advanced Spatial Analysis, University College, London, http://www.casa.ucl.ac.uk/working_papers/Paper43.pdf

Ratti C, 2002 Urban Analysis for Environmental Prediction unpublished PhD Dissertation, Department of Architecture, University of Cambridge, Cambridge

Richardson L F, 1961, "The problem of contiguity: an appendix to "The Statistics of Deadly Quarrels" "General Systems Yearbook $6139-187$

Tandy C R V, 1967, "The isovist method of landscape survey", in Symposium on Methods of Landscape Analysis Ed. H C Murray (Landscape Research Group, London) pp 9-10

Turner A, 2001, "Depthmap: a program to perform visibility graph analysis" Proceedings, Third International Symposium on Space Syntax, Atlanta 2001 31.1-31.9, The Bartlett Graduate School, University College London

Turner A, Penn A, 1999, "Making isovists syntactic: isovist integration analysis", in Proceedings, Second International Symposium on Space Syntax, Brazilia 1999 http://www.spacesyntax.net/ symposia/SSS2/sss2_proceedings.htm

Turner A, Doxa M, O'Sullivan D, Penn A, 2001, "From isovists to visibility graphs: a methodology for the analysis of architectural space" Environment and Planning B: Planning and Design $\mathbf{2 8}$ $103-121$

Wilson A G, 1998, 'Land use-transport interaction models: past and future" Journal of Transport Economics and Policy 32 -26 